\title{
Spin memory and spin-lattice relaxation in two-dimensional hexagonal crystals
}

\author{
H. Ochoa, ${ }^{1}$ F. Guinea, ${ }^{1}$ and V. I. Fal'ko ${ }^{2}$ \\ ${ }^{1}$ Instituto de Ciencia de Materiales de Madrid, CSIC, Sor Juana Inés de la Cruz 3, 28049 Madrid, Spain \\ ${ }^{2}$ Physics Department, Lancaster University, Lancaster LAI 4YB, United Kingdom \\ (Received 5 August 2013; revised manuscript received 21 September 2013; published 15 November 2013)
}

\begin{abstract}
We propose a theory of spin relaxation of electrons and holes in two-dimensional hexagonal crystals such as atomic layers of transition-metal dichalcogenides $\left(\mathrm{MoS}_{2}, \mathrm{WSe} 2\right.$, etc.). We show that, even in intrinsically defect-free crystals, their flexural deformations are able to generate spin relaxation of carriers. Based on symmetry analysis, we formulate a generic model for spin-lattice coupling between electrons and flexural deformations and use it to determine temperature- and material-dependent spin lifetimes in atomic crystals in ambient conditions.
\end{abstract}

DOI: 10.1103/PhysRevB.88.195417

PACS number(s): 73.23.-b, 75.70.Tj, 85.75.-d

\section{INTRODUCTION}

Atomically thin crystals, derived by exfoliation from van der Waals-coupled layered materials, ${ }^{1}$ represent very natural and truly two-dimensional (2D) systems for implementation in electronic devices. Starting with graphene, ${ }^{2,3}$ the family of atomically thin $2 \mathrm{D}$ crystals already includes silicene, ${ }^{4,5}$ graphane $\left(\mathrm{C}_{2} \mathrm{H}_{2}\right),{ }^{6}$ gemanane $\left(\mathrm{Ge}_{2} \mathrm{H}_{2}\right),{ }^{7}$ monolayers of hexagonal boron nitride $(\mathrm{hBN}){ }^{8}$ transition-metal dichalcogenides $M X_{2}(M=\mathrm{Mo}, \mathrm{W}, \mathrm{Ta} ; X=\mathrm{S}, \mathrm{Se}, \mathrm{Te}),{ }^{9,10}$ and bilayers of gallium chalcogenides $\left(\mathrm{Ga}_{2} X_{2}\right) \cdot{ }^{11}$ In spite of different chemical compositions, these crystals share a common honeycomblike lattice and several similar features in their electronic properties. The gap edges in the semiconducting dichalcogenides are located at the two inequivalent corners of the hexagonal Brillouin zone, the $K$ and $K^{\prime}$ points, which are related by time-inversion symmetry. An additional symmetry, which looks like a fictitious time-reversal symmetry restricted to each valley, can be defined. ${ }^{12-16}$ For the electron orbital dynamics, such fictitious time-reversal symmetry breaking can be associated with the appearance of a valley-antisymmetric pseudomagnetic field in deformed graphene,${ }^{16}$ or with trigonal warping of the electron dispersion. ${ }^{15,17}$

Also, a fictitious time-reversal symmetry breaking can be associated with the opposite sign of the spin-orbital (SO) splitting for the electron states in $\pm K$ valleys which is stronger in materials with heavier $X$ and $M$ elements. ${ }^{18,19}$ Another specific feature of SO coupling in two-dimensional hexagonal crystals (2DHCs) arises from their $z \rightarrow-z$ mirror-reflection symmetry. Since the in-plane components $s_{x, y}$ of the electron spin invert their sign upon $z \rightarrow-z$ reflection, only $s_{z}$ appears in generic $\mathbf{k} \cdot \mathbf{p}$ Hamiltonians for electrons, ${ }^{20-24}$

$$
\mathcal{H}( \pm \mathbf{K}+\mathbf{p})=\mathcal{H}_{\text {band }}( \pm \mathbf{K}+\mathbf{p})+\epsilon_{z}(\mathbf{p}) \hat{\mathcal{L}}_{z} \hat{s}_{z},
$$

where $\mathbf{p}$ is the electron momentum counted from the center of the valley. Here, the first term describes the electron/hole orbital motion in the band: $\mathcal{H}_{\text {band }} \approx \mathbf{p}^{2} / 2 m_{*}$ for gapped 2DHCs, in contrast to the linear dispersion of electrons in graphene and silicene, where $\mathcal{H}_{\text {band }} \approx v \mathbf{p} \sigma$ with Pauli matrices $\sigma_{x, y, z}$ acting on the sublattice component of the electronic wave function. The second term in Eq. (1) takes into account the atomic SO coupling, where the microscopic form of the angular momentum operator, $\hat{\mathcal{L}}_{z}= \pm 1$ for $\pm K$ in $M X_{2}$ and $\hat{\mathcal{L}}_{z}= \pm \sigma_{z}$ in graphene and silicene, and the size of the coupling constant, $\epsilon_{z}(\mathbf{p}) \approx \epsilon_{z}+p^{2} \delta m_{*}^{-1}$, depends on the material and band-specific orbital composition of the electron Bloch states. ${ }^{17,25,26}$ In each of the two valleys $\pm K$, this produces a spin splitting, $\left|\epsilon_{z}\right|$, for electrons (holes), separating their constant-energy contours in the momentum space by $\epsilon_{z} / v$, where $v(\varepsilon)$ is the electron velocity $\hat{\mathbf{v}}=\partial H_{\text {band }} / \partial \mathbf{p}$ at energy $\varepsilon$.

For all 2DHCs the $s_{z}$ component of the electron spin conserves, resulting in a long spin memory. ${ }^{27-29}$ This hints that 2DHCs offer a promising materials platform for spintronics applications. A long spin memory of photoexcited electrons and holes, at the time scale of several nanoseconds, has been already observed ${ }^{27}$ in $\mathrm{MoS}_{2}$. At the same time, it has been noticed ${ }^{30-33}$ that out-of-plane (flexural) deformations of graphene locally break its $z \rightarrow-z$ mirror symmetry and couple the in-plane $s_{x, y}$ spin components of the electron to its lateral orbital motion. A similar effect can generate spin relaxation of charge carriers in 2DHC-based spintronic devices free of intrinsic structural defects. In this paper, we study how out-of-plane spin relaxation is generated by spinlattice coupling with flexural phonons or substrate-induced bending of 2DHCs. This study is based on the symmetry analysis of possible spin-lattice couplings permissible in weakly perturbed $2 \mathrm{DHCs}$, and it is used to identify relevant parametric regimes of spin relaxation of electrons and holes.

The manuscript is organized as follows. In Sec. II we present the possible spin-lattice couplings in bent 2DHCs. We consider the geometrical coupling generated by the lack of a global normal direction to the 2DHC plane, and the additional contribution from the modification of the orbital composition of the electronic states. We distinguish between long- and short-wavelength flexural deformations, determined by the length scale defined by $\epsilon_{z}$, and show that in the case of long-wavelength deformations the effect of the former coupling can be added to the latter by means of a non-Abelian gauge transformation. In Sec. III we discuss spin relaxation due to these spin-lattice couplings. Both long- and shortwavelength deformations contribute to spin relaxation assisted by external disorder, and also short-wavelength deformations permit electronic scattering between isoenergetic states with opposite spin polarization, which constitutes an additional source of spin relaxation. This analysis is applied to the cases of static wrinkles and flexural phonons. Finally, in Sec. IV we 


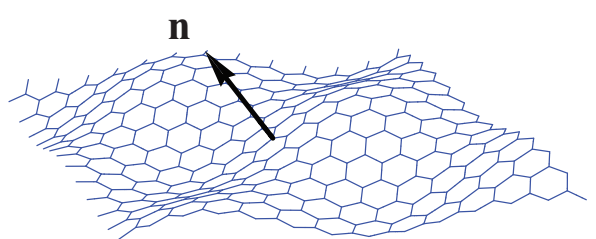

FIG. 1. (Color online) Geometry of local spin-coordinate system of electrons in wrinkled 2DHCs.

perform numerical estimates for the spin-relaxation times in experimentally relevant situations for monolayer $\mathrm{MoS}_{2}$.

\section{SPIN-LATTICE COUPLING}

Spin-lattice coupling between electrons and wrinkles can be incorporated in Eq. (1) by treating the $2 \mathrm{DHC}$ as a flexible membrane described by local vertical displacement $h(x, y)$ and local normal vector $\mathbf{n}=\left(-\partial_{x} h,-\partial_{y} h, 1\right)$; see Fig. 1 . Then, we use the global coordinate system for the three-dimensional (3D) electron spin $\mathbf{s}$ to write down its coupling to the local angular momentum oriented along $\mathbf{n}(x, y)$,

$$
\begin{array}{r}
H_{\mathrm{SO}}=\epsilon_{z} \hat{\mathcal{L}}_{z} \hat{\mathbf{s}} \cdot \mathbf{n} \approx \epsilon_{z} \hat{\mathcal{L}}_{z} \hat{s}_{z}+\delta H_{g} ; \\
\delta H_{g}=-\epsilon_{z} \hat{\mathcal{L}}_{z}\left(\partial_{x} h \hat{s}_{x}+\partial_{y} h \hat{s}_{y}\right) .
\end{array}
$$

Here, the inhomogeneous term $\delta H_{g}$ is responsible for spinlattice relaxation, whereas the first term $\epsilon_{z} \hat{\mathcal{L}}_{z} s_{z}$ sets a global quantization axis for the electron spin. Additionally, we take into account contributions towards spin-phonon coupling arising from the modification of the orbital composition of the Bloch states of electrons, due to the mixing of bands (and corresponding atomic orbitals) by mutual displacements of atoms in the 2DHC lattice. Such couplings depend on the local curvature tensor $h_{i j}^{\prime \prime} \equiv \partial_{i} \partial_{j} h$ of the deformed 2DHC rather than $\mathbf{n},{ }^{30,32}$ since tilting of a crystal does not change the orbital composition of electronic states. Phenomenologically, such additional couplings,

$$
\begin{aligned}
\delta H_{o}= & \lambda_{\|}\left[2 h_{x y}^{\prime \prime} \hat{s}_{x}+\left(h_{y y}^{\prime \prime}-h_{x x}^{\prime \prime}\right) \hat{s}_{y}\right] \hat{\mathcal{L}}_{z}+\hbar \beta(\mathbf{v} \times \mathbf{s})_{z} \nabla^{2} h \\
& +\hbar \tilde{\beta}\left[\left(\hat{v}_{x} \hat{s}_{y}+\hat{v}_{y} \hat{s}_{x}\right)\left(h_{x x}^{\prime \prime}-h_{y y}^{\prime \prime}\right)+\left(\hat{v}_{y} \hat{s}_{y}-\hat{v}_{x} \hat{s}_{x}\right) 2 h_{x y}^{\prime \prime}\right],
\end{aligned}
$$

appear as invariants of the symmetry group of a $2 \mathrm{DHC}$, built from the components of the electron spin operator $s_{x, y}$, the velocity operator, and the curvature tensor. The corresponding 2DHC symmetry group includes lattice translations, amended by $C_{6 v}$ rotations and reflections for graphene and by $D_{3 h}=$ $D_{3} \times \sigma_{h}$ for $M X_{2}$ and $\mathrm{Ga}_{2} X_{2}$. Table I lists irreducible representations (IrReps) of $D_{3 h}$ (for $C_{6 v}$ see Ref. 32) and classifies relevant operators with respect to their transformation properties, including $z \rightarrow-z$ mirror reflection and time inversion. Consequently, all terms in Eq. (3) are scalar products of operators belonging to IrReps in Table I. Note that the terms in the first row of Eq. (3) can be written as $\lambda_{\|} \hat{\mathcal{L}} \cdot \hat{\mathbf{s}}$, where $\hat{\mathcal{L}}= \pm\left(2 \partial_{x} \partial_{y} h, \partial_{y}^{2} h-\partial_{x}^{2} h\right)$ for $\pm K$ has the properties of an in-plane component of an atomic angular momentum operator, and the other two terms are similar to Bychkov-Rashba ${ }^{34,35}$ and Dresselhaus ${ }^{36}$ SO coupling. ${ }^{37}$ In those 2DHC bands where electrons originate from atomic $P^{x, y}$ and $D^{x y, x^{2}-y^{2}}$ orbitals
TABLE I. Left: Classification of the electronic operators and flexural displacements according to their transformation properties under time reversal $t \rightarrow-t$ and symmetry operations of the 2DHCs without inversion symmetry. Operators belonging to representations

\begin{tabular}{|c|c|c|c|}
\hline IrRep & $D_{3 h}=D_{3} \times \sigma_{h}$ & Material & $\epsilon_{z}(\mathrm{meV})$ \\
\hline$A_{1}^{\prime}$ & $\mathcal{L}_{z} \hat{s}_{z}$ & $\mathrm{e}-\mathrm{MoS}_{2}$ & 3 \\
\hline$A_{2}^{\prime}$ & \multicolumn{3}{|c|}{$\mathcal{L}_{z}, \hat{s}_{z}$} \\
\hline$A_{1}^{\prime \prime}$ & $\hat{\mathbf{v}} \cdot \hat{\mathbf{s}}$ & h-MoS 2 & 140 \\
\hline$A_{2}^{\prime \prime}$ & $\nabla^{2} h,(\hat{\mathbf{v}} \times \hat{\mathbf{s}})_{z}$ & & \\
\hline$E^{\prime}$ & & $\mathrm{h}-\mathrm{WS}_{2}$ & 430 \\
\hline$E^{\prime \prime}$ & $\left(\begin{array}{c}\partial_{x}^{2} h-\partial_{y}^{2} h \\
2 \partial_{x} \partial_{y} h\end{array}\right)$ & h-MoSe 2 & 180 \\
\hline & $\left(\begin{array}{l}\hat{v}_{x} \hat{s}_{y}+\hat{v}_{y} \hat{s}_{x} \\
\hat{v}_{y} \hat{s}_{y}-\hat{v}_{x} \hat{s}_{x}\end{array}\right)$ & $\mathrm{h}-\mathrm{WSe}_{2}$ & 460 \\
\hline$t \rightarrow-t$ & even & & \\
\hline
\end{tabular}
$A_{1,2}^{\prime}$ and $E^{\prime}$ are $z \rightarrow-z$ even, and those belonging to $A_{1,2}^{\prime \prime}$ and $E^{\prime \prime}$ are odd. Right: SO splitting in the conduction (e) and valence (h) bands in 2DCHs of $M X_{2} \cdot{ }^{17-19}$

(e.g., valence band in $\mathrm{MoS}_{2}$ ), the influence of $\delta H_{o}$ should be less than that of $\delta H_{g}$. In the bands where electrons originate from $S, P^{z}$, or $D^{z^{2}}$ orbitals, SO coupling arises from their weak mixing with high-energy orbitals, and hence $\delta H_{g}$ and $\delta H_{o}$ should be treated on equal footing.

As they stand in Eqs. (2) and (3), the spin-lattice coupling terms $\delta H_{g, o}$ can be used to evaluate the rate of spin relaxation of electrons due to the short-wavelength ripples with a Fourier spectrum $h_{q}$ in the range of wave numbers $q \gg \epsilon_{z} / v$. To describe the spin of electrons flying across such shortwavelength ripples, we use a spin-coordinate frame related to the median orientation of the $2 \mathrm{DHC}$, averaged over many ripples periods. In contrast, it is more practical to analyze the influence of long-wavelength wrinkles, with $q<\epsilon_{z} / v$, in the local, adiabatically varying spin frame, adjusted to the local flake orientation. The electron spinor states in the global and local frames are related by the non-Abelian gauge transformation $\hat{U}=e^{\frac{i}{2} \nu \cdot \hat{\mathbf{s}}}, \quad v=\left(\partial_{y} h,-\partial_{x} h\right)$, which diagonalizes Eq. (2) into $H_{\mathrm{SO}}=\epsilon_{z} \hat{\mathcal{L}}_{z} \hat{s}_{z}$ but also produces an additional smaller perturbation,

$$
\begin{aligned}
\hat{U} \mathcal{H}_{\text {band }}( \pm \mathbf{K}+\mathbf{p}) \hat{U}^{\dagger} \approx & \mathcal{H}_{\text {band }}( \pm \mathbf{K}+\mathbf{p})+\delta \tilde{H}_{g} \\
\delta \tilde{H}_{g}= & \frac{1}{2}\left\{\frac{\partial \mathcal{H}_{\text {band }}}{\partial \mathbf{p}}, \hat{U}(-i \hbar \partial) \hat{U}^{\dagger}\right\} \\
= & \frac{\hbar}{2}\left[\hat{v}_{y} \hat{s}_{x} \partial_{y}^{2} h-\hat{v}_{x} \hat{s}_{y} \partial_{x}^{2} h\right. \\
& \left.+\left(\hat{v}_{x} \hat{s}_{x}-\hat{v}_{y} \hat{s}_{y}\right) \partial_{x} \partial_{y} h\right] .
\end{aligned}
$$

Note that, upon gauge transformation $\hat{U}$, spin-lattice coupling $\delta H_{o}$ remains almost unchanged (only terms in the higher order in $q h_{q}$ can appear), hence $\delta \tilde{H}_{g}$ is the leading term in the Taylor expansion of $\hat{U} \mathcal{H}_{\text {band }} \hat{U}^{\dagger}-\mathcal{H}_{\text {band }}$ in small $q h_{q}$. Also, $\delta \tilde{H}_{g}$ has the same structure as a combination of Bychkov-Rashba and Dresselhaus terms in the phenomenological Eq. (3), with the universal coupling constants $\beta=\tilde{\beta}=-1 / 4$, which manifests the geometrical origin of this coupling. Therefore, in the 


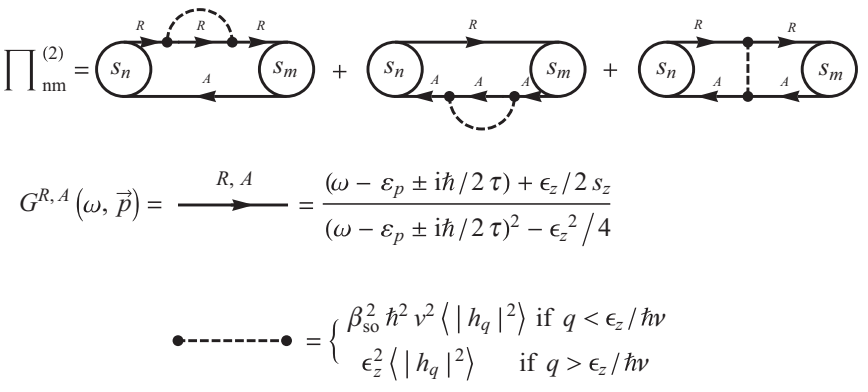

FIG. 2. The three diagrams which contribute to the $\Pi$ operator to the lowest order in the spin-lattice coupling.

following analysis of spin relaxation induced by smooth ripples, we combine $\delta \tilde{H}_{g}$ and $\delta H_{o}$ by redefining the coupling constants $\beta$ and $\tilde{\beta}$.

\section{SPIN-LATTICE RELAXATION}

Spin-lattice relaxation of electrons is determined by the cumulative contribution of both short- and long-wavelength lattice deformations, which produces the sum, $\tau_{s}^{-1}=\tau_{d}^{-1}+$ $\tau_{b}^{-1}$, of "diffusive" spin relaxation assisted by external charge disorder in the substrate and "ballistic" contribution determined by a simultaneous momentum $\left|\mathbf{p}-\mathbf{p}^{\prime}\right| \sim q$ and spin transfer to the ripples.

The diffusive contribution to the spin relaxation rate can be estimated using the framework of diagrammatic perturbation theory applied to the analysis of a disorder-averaged spindensity matrix of electrons, $\frac{1}{2} \vec{\rho} \cdot \vec{\sigma}$. Diagrammatically, in Fig. 2, spin-lattice coupling is incorporated in the polarization operator $\Pi$, which governs spin diffusion, $\left[\partial_{t}-\Pi\right] \vec{\rho}(t)=$ $\vec{\rho}(0) \delta(t)$. Without spin-lattice coupling, $\Pi \approx D \nabla^{2}$, where $D=\frac{1}{2} v^{2} \tau=\frac{1}{2} \ell v, \tau$, and $\ell$ stand for the electron diffusion coefficient, the scattering time, and the mean free path, respectively. Valley-dependent spin splitting, $\pm \epsilon_{z} s_{z}$, generates independent precession of $\vec{\rho}_{ \pm}$in $\pm K$ valleys. Spin-lattice relaxation of electrons, assisted by disorder, is incorporated into $\Pi$ via three diagrams (see Fig. 2), where solid lines indicate the free-electron Green's functions, the dashed lines are correlators $\left\langle h(\mathbf{r}) h\left(\mathbf{r}^{\prime}\right)\right\rangle$, and dots are spin-lattice coupling vertices corresponding to Eqs. (2) and (3). The kinetics of spin polarization of carriers is then described by

$$
\left[\partial_{t}-D \nabla^{2}\right] \vec{\rho}_{ \pm} \pm \lambda_{Z_{2}} \mathbf{n}_{z} \times \vec{\rho}_{ \pm}+\tau_{d}^{-1} \vec{\rho}=\vec{\rho}_{ \pm}(0) \delta(t),
$$

where \pm identifies the $\pm K$ valley, and we have neglected the difference between the in-plane and out-of-plane spinrelaxation rates, regarding the fact that, in all possible regimes, it should be superseded by a faster spin precession due to the $\epsilon_{z}$ term in Eq. (1). The three diagrams in Fig. 2 lead to ${ }^{38}$

$$
\begin{aligned}
\tau_{d}^{-1} & =\sum_{\mathbf{q}} \mathcal{M}(q) \frac{\tau q^{2}}{N}\left\langle\left|h_{\mathbf{q}}\right|^{2}\right\rangle \times\left\{\begin{array}{ll}
q^{2} \beta_{\mathrm{SO}}^{2} v^{2} & q<\frac{\epsilon_{z}}{\hbar v} \\
\epsilon_{z}^{2} / \hbar^{2} & q>\frac{\epsilon_{z}}{\hbar v}
\end{array},\right. \\
\mathcal{M}(q) & =\frac{1+\ell^{2} q^{2}+\frac{\tau^{2} \epsilon_{z}^{2}}{\hbar^{2}}}{1+2\left(\ell^{2} q^{2}+\frac{\tau^{2} \epsilon_{z}^{2}}{\hbar^{2}}\right)+\left(\ell^{2} q^{2}-\frac{\tau^{2} \epsilon_{z}^{2}}{\hbar^{2}}\right)^{2}}, \\
\beta_{\mathrm{SO}}^{2} & \equiv \beta^{2}+\tilde{\beta}^{2}+\frac{\lambda_{\|}^{2}}{\hbar^{2} v^{2}} .
\end{aligned}
$$
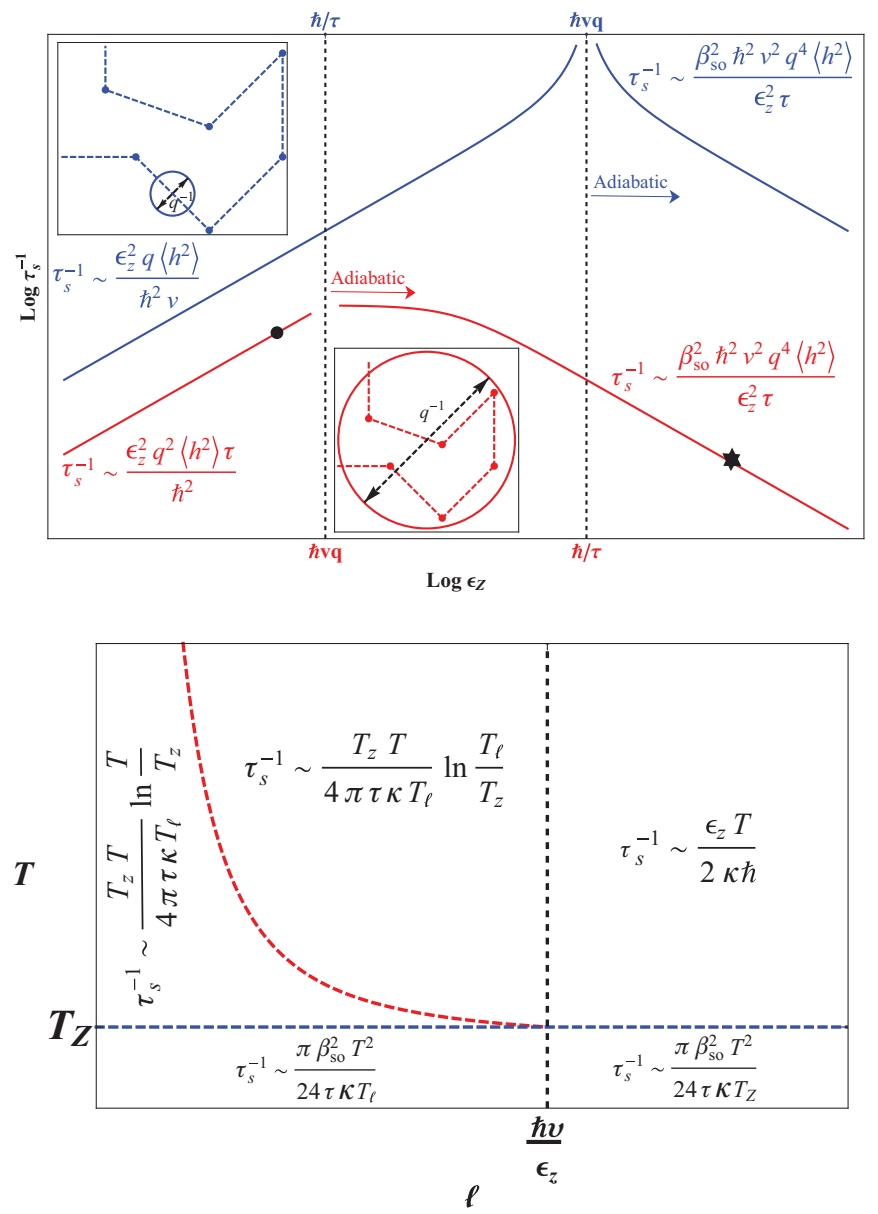

FIG. 3. (Color online) Top: Schematic behavior of spin relaxation induced by wrinkles of typical size $q^{-1}$ and height $\sqrt{\left\langle h^{2}\right\rangle}$. The top and bottom lines correspond to $q \ell>1$ and $q \ell<1$, respectively. The experimental situation ${ }^{43-46}$ for electrons and holes in $\mathrm{MoS}_{2}$ is denoted by a dot and a star, respectively. Bottom: Spin relaxation induced by flexural phonons for different regimes of temperature and disorder. The dashed line represents $T_{\ell}$.

The above expressions link together D'yakonov-Perel' ${ }^{39,40}$ and Elliot-Yafet ${ }^{41,42}$ regimes discussed in the theory of spin relaxation in disordered systems. Indeed, for $q \ell \ll 1$ and $\tau \epsilon_{z} \ll \hbar$, spin relaxation takes place over several momentumscattering events while the electron diffuses in an interval of space with almost homogeneous SO coupling which causes its spin to precess randomly (see bottom inset in Fig. 3), so spin relaxation obeys the D'yakonov-Perel' relation: ${ }^{39,40} \tau_{d}^{-1} \propto \tau$. On the contrary, for $q \ell>1$ or $\tau \epsilon_{z}>\hbar$, spin flips take place over single scattering events involving external disorder (see top inset in Fig. 3), and spin relaxation obeys the Elliot-Yaffet relation: ${ }^{41,42} \tau_{d}^{-1} \propto \tau^{-1}$.

The ballistic contribution is determined by a simultaneous momentum, $\left|\mathbf{p}-\mathbf{p}^{\prime}\right| \sim q$, and spin transfer to the lattice upon electron scattering off the ripples,

$$
\tau_{b}^{-1}=\frac{2 \pi}{N \hbar} \sum_{\mathbf{q}} e^{-\frac{1}{q^{\ell}}}\left|\left\langle\mathbf{p}+\mathbf{q} \uparrow\left|\delta H_{g}\right| \mathbf{p} \downarrow\right\rangle\right|^{2} \delta\left(\varepsilon_{\mathbf{p}+\mathbf{q}}^{\uparrow}-\varepsilon_{\mathbf{p}}^{\downarrow}\right) .
$$


Here, a factor $e^{-\frac{1}{q \ell}}$ takes into account the fact that this contribution does not involve any externally promoted momentum transfer. This contribution is generated by the shortwavelength flexural deformations $h_{q}$ with $q>\epsilon_{z} / \hbar v$, which permit electron scattering between isoenergy lines separated by $\epsilon_{z} / \hbar v$ on the momentum plane near the $\pm K$ points (the local spin quantization axis is defined as a normal to the median plane averaging 2DHC wrinkles over $\hbar v / \epsilon_{z}$ scale).

\section{A. Static wrinkles}

We analyze the spin relaxation of electrons produced by static wrinkles characterized by lateral size $q^{-1}$ and height $\sqrt{\left\langle h^{2}\right\rangle}$. When $\epsilon_{z} \gg \hbar v q, \hbar / \tau$ the ballistic contribution is absent, and $\tau_{s}$ obeys the Elliot-Yafet relation as we justified before. For short-wavelength wrinkles, however, both contributions are present in principle. As we explained before, we distinguish two asymptotic regimes characterized by the ratio $q \ell$ (see Fig. 3). In the case of $q \ell>1$ we expect an Elliot-Yafet behavior for the diffusive contribution. Moreover, for $\epsilon_{z}<\hbar v q, \tau_{b}^{-1} \gg \tau_{d}^{-1}$. However, for $q \ell \ll 1, \tau_{b}^{-1}$ is exponentially suppressed, and $\tau_{s}$ obeys the D'yakonov-Perel' relation. In both cases we find a nonmonotonic dependence of $\tau_{s}^{-1}$ on $\epsilon_{z}$, due to the double role it plays: on the one hand, $\epsilon_{z}$ determines the strength of the spin-lattice coupling according to Eq. (2), and on the other, it represents the intrinsic SO splitting leading to a pseudo-Zeeman field which protects spin polarization.

\section{B. Flexural phonons}

Spin relaxation due to flexural phonons is evaluated taking into account that their quadratic dispersion $\omega_{\mathbf{q}}=\sqrt{\frac{\kappa}{\rho}}|\mathbf{q}|^{2}$ (where $\kappa$ is the bending rigidity of the system and $\rho=M / A_{c}$ the mass density) and the resulting low frequencies allow us to treat them as quasistatic deformations parametrized by spectral density $\left\langle\left|h_{\mathbf{q}}\right|^{2}\right\rangle$,

$$
\left\langle\left|h_{\mathbf{q}}\right|^{2}\right\rangle=\frac{\hbar}{2 M \omega_{\mathbf{q}}}\left[2 n_{B}\left(\frac{\omega_{\mathbf{q}}}{T}\right)+1\right],
$$

where $n_{B}$ is the Bose-Einstein distribution function. For the sake of convenience, we introduce two characteristic temperature scales associated to $\ell$ and $\epsilon_{z}:{ }^{47} T_{\ell} \equiv \ell^{-2} \hbar \sqrt{\kappa / \rho}$ and $T_{Z} \equiv \sqrt{\kappa / \rho} \epsilon_{z}^{2} / \hbar v^{2}$. For $T \ll T_{Z}$, spin relaxation is dominated by long-wavelength $\left(q<\epsilon_{z} / \hbar v\right)$ phonons, whereas at $T \gg T_{Z}$, short-wavelength modes dominate. On the other hand, at $T<T_{\ell}$ only modes with $q<\ell^{-1}$ contribute to spin relaxation. At low temperatures D'yakonov-Perel' behavior is expected if $T_{\ell}>T_{Z}$, whereas for $T_{\ell}<T_{Z}$ the relation $\tau_{s} \propto \tau$ is anticipated. At high temperatures, however, the analysis is not so straightforward since also the ballistic contribution must be taken into account. Two regimes of disorder are defined by the ratio $T_{Z} / T_{\ell}$, which controls the relative importance of both contributions as is shown next.

The dependence on temperature of $\tau_{d}$ can be easily inferred from Eq. (6). The integration over flexural phonon modes ${ }^{48}$ can be expressed as

$$
\begin{aligned}
\tau_{d}^{-1}= & \frac{\beta_{\mathrm{SO}}^{2} T^{2}}{8 \pi \tau \kappa T_{\ell}} \int^{\frac{T_{Z}}{T}} d x x \mathcal{M}(x)\left[2 n_{B}(x)+1\right] \\
& +\frac{\tau \epsilon_{z}^{2} T}{8 \pi \hbar^{2} \kappa} \int_{\frac{T_{Z}}{T}} d x \mathcal{M}(x)\left[2 n_{B}(x)+1\right], \\
\mathcal{M}(x)= & \frac{1+\frac{T}{T_{\ell}} x+\frac{T_{Z}}{T_{\ell}}}{1+2\left(\frac{T}{T_{\ell}} x+\frac{T_{Z}}{T_{\ell}}\right)+\left(\frac{T}{T_{\ell}} x-\frac{T_{Z}}{T_{\ell}}\right)^{2}},
\end{aligned}
$$

where we distinguish the contributions from long- and shortwavelength modes, respectively. After the integration over thermally excited flexural phonon modes, we find

$$
\tau_{d}^{-1} \approx \begin{cases}\frac{\pi \beta_{\mathrm{SO}}^{2} T^{2}}{24 \tau \kappa T_{\ell}} & T \ll T_{Z} \ll T_{\ell}, \\ \frac{T_{Z} T}{4 \pi \tau T_{\ell}} \ln \left(\frac{T}{T_{Z}}\right) & T_{Z} \ll T \ll T_{\ell}, \\ \frac{T_{Z} T}{4 \pi \tau \kappa T_{\ell}} \ln \left(\frac{T_{\ell}}{T_{Z}}\right) & T_{Z} \ll T_{\ell} \ll T, \\ \frac{\pi \beta_{S O}^{2} T^{2}}{24 \tau \kappa T_{Z}} & T_{\ell}, T \ll T_{Z}, \\ \frac{T}{4 \pi \kappa \tau} & T_{\ell} \ll T_{Z} \ll T .\end{cases}
$$

Among all these regimes, only the asymptotic of $T \gg T_{Z}$ and $\hbar v / \epsilon_{z}>\ell$ is dominated by ballistics,

$$
\begin{aligned}
\tau_{b}^{-1} & =\frac{2 \varepsilon T_{Z}}{\hbar \kappa} f\left(\frac{T_{M}}{T}, \frac{T_{m}}{T}, \frac{T_{\ell}}{T}\right), \\
f(X, x, z) & \equiv \int_{x}^{X} \frac{d y}{2 \pi} \frac{\frac{e^{y}+1}{e^{y}-1} e^{-\sqrt{z / y}}}{\sqrt{(y-x)(X-y)}}, \\
T_{M, m} & =\frac{4 \varepsilon T_{Z}}{\epsilon_{z}}\left(\sqrt{\varepsilon / \epsilon_{z}} \pm \sqrt{\varepsilon / \epsilon_{z}-1}\right)^{2} .
\end{aligned}
$$

When $z \ll X, x$ we have

$$
f \approx \begin{cases}\sqrt{\frac{1}{X x}} & X, x \ll 1, \\ \sqrt{\frac{1}{\pi X}} e^{-x} \operatorname{erf}(\sqrt{X-x}) & X, x \gg 1 .\end{cases}
$$

If $z \gg X, x$ then $f$ is exponentially suppressed. For $T>T_{M}>$ $T_{\ell}$, we find that $\frac{\tau_{b}^{-1}}{\tau_{d}^{-1}} \sim 2 \pi \frac{\tau \epsilon_{z}}{\hbar} \gg 1$; otherwise the ballistic contribution is exponentially small and the diffusive one dominates. Thus, having compared both contributions and combined them in $\tau_{s}^{-1}=\tau_{d}^{-1}+\tau_{b}^{-1}$, we summarize the resulting behaviors in Fig. 3 .

\section{DISCUSSION}

In currently available $\mathrm{MoS}_{2}$-based devices, the mobilities extracted from transport experiments ${ }^{44-46}$ indicate that the diffusive contribution limits spin lifetimes of electrons and holes. The experimental situation in both cases is indicated in Fig. 3. For wrinkles with a typical height of $1 \mathrm{~nm}$ and lateral length scale of $10 \mathrm{~nm}$, as reported in Ref. 43 and in the SO splitting mentioned in Table I, we find ${ }^{49} \tau_{s} \sim 1 \mathrm{~ns}$. For the same conditions we estimate the spin lifetime of the holes as $\tau_{s} \geqslant 10 \mathrm{~ns}$.

For perfectly flat $\mathrm{MoS}_{2}$, flexural vibrations thermally activated at room temperature would also produce spin 
relaxation. ${ }^{50}$ We estimate the bending rigidity of a single layer,

$$
\kappa=\frac{Y \delta^{3}}{24\left(1-\sigma^{2}\right)} \approx 27 \mathrm{eV},
$$

by describing an $\mathrm{MoS}_{2}$ crystal as a plate of thickness $\delta \approx$ $6.75 \AA$, where $\delta$ is the interlayer distance in bulk $\mathrm{MoS}_{2} .{ }^{51} \mathrm{In}$ Eq. (11), $Y=0.33 \mathrm{TPa}$ is the Young modulus ${ }^{52}$ and $\sigma=0.125$ is the Poisson ratio. ${ }^{53}$ Flexural phonons at room temperature limit the spin lifetime of electrons by ${ }^{54} \tau_{s} \sim 5 \mathrm{~ns}$ and that for holes by $\tau_{s} \sim 20 \mathrm{~ns}$. The latter estimate is produced without taking into account that an atomically flat substrate will quench bending modes ${ }^{55}$ so that we expect $2 \mathrm{DHCs}-\mathrm{hBN}$ structures to exhibit a longer spin memory of charge carriers and therefore offer a perfect platform for spintronics devices.

\section{ACKNOWLEDGMENTS}

We thank T. Heinz, A. Morpurgo, R. Roldán, X. Xu, and V. Zólyomi for useful discussions. This study was funded by a JAE-Pre grant (CSIC, Spain); MINECO, Spain, through Grant No. FIS2011-23713; ERC Advanced Grant No. 290846 and "Graphene and Beyond," ERC Synergy Grant "Hetero2D," and the European Graphene Flagship Project.
${ }^{1}$ A. K. Geim and I. V. Grigorieva, Nature (London) 499, 419 (2013).

${ }^{2}$ K. S. Novoselov, A. K. Geim, S. V. Morozov, D. Jiang, Y. Zhang, S. V. Dubonos, I. V. Grigorieva, and A. A. Firsov, Science 306, 666 (2004).

${ }^{3}$ A. H. C. Neto, F. Guinea, N. M. R. Peres, K. S. Novoselov, and A. K. Geim, Rev. Mod. Phys. 81, 109 (2009).

${ }^{4}$ P. Vogt, P. De Padova, C. Quaresima, J. Avila, E. Frantzeskakis, M. C. Asensio, A. Resta, B. Ealet, and G. Le Lay, Phys. Rev. Lett. 108, 155501 (2012).

${ }^{5}$ A. Fleurence, R. Friedlein, T. Ozaki, H. Kawai, Y. Wang, and Y. Yamada-Takamura, Phys. Rev. Lett. 108, 245501 (2012).

${ }^{6}$ D. C. Elias, R. R. Nair, T. M. G. Mohiuddin, S. V. Morozov, P. Blake, M. P. Halsall, A. C. Ferrari, D. W. Boukhvalov, M. I. Katsnelson, A. K. Geim et al., Science 323, 610 (2009).

${ }^{7}$ E. Bianco, S. Butler, S. Jiang, O. D. Restrepo, W. Windl, and J. E. Goldberger, ACS Nano 7, 4414 (2013).

${ }^{8}$ K. Watanabe, T. Taniguchi, and H. Kanda, Nat. Mater. 3, 404 (2004). ${ }^{9}$ R. A. Gordon, D. Yang, E. D. Crozier, D. T. Jiang, and R. F. Frindt, Phys. Rev. B 65, 125407 (2002).

${ }^{10}$ Q. H. Wang, K. Kalantar-Zadeh, A. Kis, J. N. Coleman, and M. S. Strano, Nat. Nanotechnol. 7, 699 (2012).

${ }^{11}$ V. Zólyomi, N. D. Drummond, and V. I. Fal'ko, Phys. Rev. B 87, 195403 (2013).

${ }^{12}$ S. Iordanskii and A. E. Koshelev, JETP Lett. 41, 574 (1985).

${ }^{13}$ A. F. Morpurgo and F. Guinea, Phys. Rev. Lett. 97, 196804 (2006).

${ }^{14}$ E. McCann, K. Kechedzhi, V. I. Fal'ko, H. Suzuura, T. Ando, and B. L. Altshuler, Phys. Rev. Lett. 97, 146805 (2006).

${ }^{15}$ K. Kechedzhi, V. I. Fal'ko, E. McCann, and B. L. Altshuler, Phys. Rev. Lett. 98, 176806 (2007).

${ }^{16}$ M. A. H. Vozmediano, M. I. Katsnelson, and F. Guinea, Phys. Rep. 496, 109 (2010).

${ }^{17}$ A. Kormányos, V. Zólyomi, N. D. Drummond, P. Rakyta, G. Burkard, and V. I. Fal'ko, Phys. Rev. B 88, 045416 (2013).

${ }^{18}$ Z. Y. Zhu, Y. C. Cheng, and U. Schwingenschlögl, Phys. Rev. B 84, 153402 (2011).

${ }^{19}$ W. Feng, Y. Yao, W. Zhu, J. Zhou, W. Yao, and D. Xiao, Phys. Rev. B 86, 165108 (2012).

${ }^{20}$ C. L. Kane and E. J. Mele, Phys. Rev. Lett. 95, 226801 (2005).

${ }^{21}$ D. Huertas-Hernando, F. Guinea, and A. Brataas, Phys. Rev. B 74, 155426 (2006).

${ }^{22}$ H. Min, J. E. Hill, N. A. Sinitsyn, B. R. Sahu, L. Kleinman, and A. H. MacDonald, Phys. Rev. B 74, 165310 (2006).
${ }^{23}$ D. Xiao, G.-B. Liu, W. Feng, X. Xu, and W. Yao, Phys. Rev. Lett. 108, 196802 (2012).

${ }^{24}$ H. Ochoa and R. Roldán, Phys. Rev. B 87, 245421 (2013).

${ }^{25}$ H. Rostami, A. G. Moghaddam, and R. Asgari, Phys. Rev. B 88, 085440 (2013).

${ }^{26}$ E. Cappelluti, R. Roldán, J. A. Silva-Guillén, P. Ordejón, and F. Guinea, Phys. Rev. B 88, 075409 (2013).

${ }^{27}$ K. F. Mak, K. He, J. Sahn, and T. F. Heinz, Nat. Nanotechnol. 7, 494 (2012).

${ }^{28}$ H. Zeng, J. Dai, W. Yao, D. Xiao, and X. Cui, Nat. Nanotechnol. 7, 490 (2012).

${ }^{29}$ T. Cao, G. Wang, W. Han, H. Ye, C. Zhu, J. Shi, Q. Niu, P. Tan, E. Wang, B. Liu et al., Nat. Commun. 3, 887 (2012).

${ }^{30}$ J.-S. Jeong, J. Shin, and H.-W. Lee, Phys. Rev. B 84, 195457 (2011).

${ }^{31}$ E. McCann and V. I. Fal'ko, Phys. Rev. Lett. 108, 166606 (2012).

${ }^{32}$ H. Ochoa, A. H. Castro Neto, V. I. Fal'ko, and F. Guinea, Phys. Rev. B 86, 245411 (2012).

${ }^{33}$ S. Fratini, D. Gosálbez-Martínez, P. M. Cámara, and J. FernándezRossier, Phys. Rev. B 88, 115426 (2013).

${ }^{34}$ Y. A. Bychkov and E. I. Rashba, J. Phys. C 17, 6039 (1984).

${ }^{35}$ E. I. Rashba, Phys. Rev. B 79, 161409(R) (2009).

${ }^{36}$ G. Dresselhaus, Phys. Rev. 100, 580 (1955).

${ }^{37}$ Note that there is another possible combination of velocity and spin operators which can be written as $\hat{\mathbf{v}} \cdot \hat{\mathbf{s}}$, but this is a complete pseudoscalar which cannot be coupled with these excitations.

${ }^{38}$ Note that here the height profile reads $h(\mathbf{x})=\frac{1}{\sqrt{N}} \sum_{\mathbf{q}} h_{\mathbf{q}} e^{i \mathbf{q} \cdot \mathbf{x}}$, where $N$ is the number of unit cells of the system. Therefore, the Fourier components $h_{\mathbf{q}}$ have also dimensions of length.

${ }^{39}$ M. I. D'yakonov and V. I. Perel', Sov. Phys. Solid State 13, 3023 (1971).

${ }^{40}$ M. I. D'yakonov, Spin Physics in Semiconductors (Springer, Berlin, 2008).

${ }^{41}$ P. G. Elliot, Phys. Rev. 96, 266 (1954).

${ }^{42}$ Y. Yafet, Solid State Physics (Academic, New York, 1963).

${ }^{43}$ J. Brivio, D. T. L. Alexander, and A. Kis, Nano Lett. 11, 5148 (2011).

${ }^{44}$ B. Radisavljevic, A. Radenovic, J. Brivio, V. Giacometti, and A. Kis, Nat. Nanotechnol. 6, 147 (2011).

${ }^{45}$ Y. Zhang, J. Ye, Y. Matsuhashi, and Y. Iwasa, Nano Lett. 12, 1136 (2012).

${ }^{46}$ W. Bao, X. Cai, D. Kim, K. Sridhara, and M. S. Fuhrer, Appl. Phys. Lett. 102, 042104 (2013).

${ }^{47}$ Boltzmann constant $k_{B}$ is set to 1 . 
${ }^{48}$ After the substitution $\frac{1}{N} \sum_{\mathbf{q}} \rightarrow \frac{\mathcal{A}_{c}}{4 \pi^{2}} \int_{\mathrm{BZ}} d^{2} \mathbf{q}$, where $\mathcal{A}_{c}$ is the area of the unit cell.

${ }^{49}$ For electrons we employ the formula $\tau_{s}^{-1} \approx \frac{\epsilon_{z}^{2} q^{2}\left\langle h^{2}\right\rangle \tau}{\hbar^{2}}$, whereas for holes $\tau_{s}^{-1} \approx \frac{\beta_{\mathrm{SO}}^{2} \hbar^{2} v^{2} q^{4}\left\langle h^{2}\right\rangle}{\epsilon_{z}^{2} \tau}$, with $\beta_{\mathrm{SO}}^{2}=\frac{1}{16}$ [note that in addition the spin-phonon coupling Eq. (3) should be included in $\beta_{\mathrm{SO}}^{2}$ ].

${ }^{50}$ L. D. Landau and E. M. Lifshitz, Theory of Elasticity (Pergamon, Oxford, 1959).

${ }^{51}$ M. M. Benamen, B. Radisavljevic, J. S. Héron, S. Sahoo, H. Berger, and A. Kis, Nanotechnology 22, 125706 (2011).
${ }^{52}$ A. Castellanos-Gómez, M. Poot, G. A. Steele, H. S. J. van der Zant, N. Agraï, and G. Rubio-Bollinger, Adv. Mater. 24, 772 (2012).

${ }^{53}$ M. Lovell, M. Khonsari, and R. Marangoni, Wear 194, 60 (1996).

${ }^{54}$ For electrons we have $T_{\ell} \sim 10 \mathrm{~K}$ and $T_{Z} \sim 10^{-3} T_{\ell}$, so we employ the formula $\tau_{s}^{-1} \approx \frac{T_{Z} T}{4 \pi \tau T_{\ell}} \ln \left(\frac{T_{\ell}}{T_{Z}}\right)$. For holes, since $T_{Z} \sim 3000 \mathrm{~K}$, we employ the formula $\tau_{s}^{-1} \approx \frac{\pi \beta_{S O}^{2} T^{2}}{24 \tau \kappa T_{Z}}$.

${ }^{55} \mathrm{~B}$. Amorim and F. Guinea, Phys. Rev. B 88, 115418 (2013). 